\title{
Long-Slit Spectroscopy of Parsec-Scale Jets from DG TAuri
}

\author{
Heeyoung $\mathrm{OH}^{1,2}$, TAe-Soo Pyo ${ }^{3,4}$, In-Soo YuK ${ }^{2}$, And ByeOng-Gon PARK ${ }^{1,2}$ \\ ${ }^{1}$ Korea University of Science and Technology, 217 Gajeong-ro, Yuseong-gu, Daejeon 305-350, Korea \\ hyoh@kasi.re.kr, bgpark@kasi.re.kr \\ ${ }^{2}$ Korea Astronomy and Space Science Institute,776 Daedeokdae-ro, Yuseong-gu, Daejeon 305-348, Korea \\ yukis@kasi.re.kr \\ ${ }^{3}$ Subaru Telescope, National Astronomical Observatory of Japan, 650 North A'ohoku Place, Hilo, HI 96720, USA \\ pyo@subaru.naoj.org \\ ${ }^{4}$ School of Mathematical and Physical Science, SOKENDAI (The Graduate University for Advanced Studies), Hayama, \\ Kanagawa 240-0193, Japan
}

Received December 24, 2014; accepted March 16, 2015

\begin{abstract}
We present observational results from optical long-slit spectroscopy of parsec-scale jets of DG Tau. From HH 158 and HH 702, the optical emission lines of H $\alpha$, [O I] $\lambda \lambda 6300,6363$, [N II] $\lambda \lambda 6548$, 6584, and [S II] $\lambda \lambda 6716,6731$ are obtained. The kinematics and physical properties (i.e., electron density, electron temperature, ionization fraction, and mass-loss rate) are investigated along the blueshifted jet

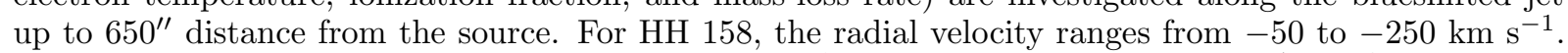
The proper motion of the knots is $0^{\prime \prime} .196-00^{\prime \prime} 272 \mathrm{yr}^{-1}$. The electron density is $\sim 10^{4} \mathrm{~cm}^{-3}$ close to the star, and decreases to $\sim 10^{2} \mathrm{~cm}^{-3}$ at $14^{\prime \prime}$ away from the star. Ionization fraction indicates that the gas is almost neutral in the vicinity of the source. It increases up to over 0.4 along the distance. HH 702 is located at $650^{\prime \prime}$ from the source. It shows $\sim-80 \mathrm{~km} \mathrm{~s}^{-1}$ in the radial velocity. Its line ratios are similar to those at knot $\mathrm{C}$ of $\mathrm{HH} 158$. The mass-loss rate is estimated to be about $\sim 10^{-7} M_{\odot} \mathrm{yr}^{-1}$, which is similar to values obtained from previous studies.
\end{abstract}

Key words: stars: formation — stars: outflows — Herbig-Haro obejcts — stars: individual: DG Tau — techniques: spectroscopy

\section{INTRODUCTION}

In the formation of stars and planetary systems, studying outflows and mass-accretion is essential (Hartigan et al. 1995). Through the analysis of the kinematics and physical conditions of outflows from young stellar objects (YSOs), we are able to understand the jet launching mechanism and the interaction with ambient material. Parsec-scale jets are useful to study star formation in somewhat long time scale and large spatial distribution. There are a number of parsec-scale jets known (e.g., Ray 1987; Bally \& Devine 1994; Ogura 1995; Bally et al. 1995). One of the extreme cases is the HH 222 system, suggested as a giant Herbig-Haro flow spread over 5.3 parsec in length (Reipurth et al. 2013).

Mundt \& Fried (1983) discovered a jet-like outflow from DG Tau. DG Tau is an early evolutional stage CTTS (Class II) with mass of $0.67 M_{\odot}$ (Hartigan et al. 1995) and age of about $3 \times 10^{5} \mathrm{yr}$ (Beckwith et al. 1990). McGroarty \& Ray (2004) suggested that it is the driving source of a parsec-scale jet including $\mathrm{HH}$ 158, HH 702, and HH 830. The later two HH objects are located at more than $9^{\prime}$ away from DG Tau. In their follow up observations, McGroarty et al. (2007) reported that HH 702 showed receding motion with respect to DG Tau. They also concluded that HH 830 is not ejected from DG Tau. Eislöffel \& Mundt (1998)

CORRESPONDING AUThOR: T.-S. Pyo showed that the HH 158 outflow extends to $\sim 11^{\prime \prime}$ toward the southwest direction and estimated the direction of the proper motion of knots from DG Tau as $\sim 226 \pm 7^{\circ}$. They also calculated the jet inclination angle as $\sim 32^{\circ}$ with respect to the line of sight. In addition to an optical jet, Rodríguez et al. (2012) and Lynch et al. (2013) detected a radio knot at $\sim 7^{\prime \prime}$ from the central source. High spatial resolution observations with a space telescope and ground based large telescope with an adaptive optics system showed that the blueshifted HH 158 jet can be traced to within $\sim 0^{\prime \prime} 1$ from the star (Bacciotti et al. 2000, 2002; Pyo et al. 2003; Maurri et al. 2014). Lavalley et al. (1997) reported that the redshifted jet with the length of $\sim 1^{\prime \prime}$. Pyo et al. (2003) showed that there is a $0^{\prime \prime} .7$ gap between the redshifted jet and the star in the [Fe II] $1.644 \mu \mathrm{m}$ emission, which indicates the presence of an optically thick disk. The two velocity components are shown in the optical and near infrared forbidden emission lines (Hartmann \& Raymond 1989; Solf \& Böhm 1993; Hamann 1994; Hartigan et al. 1995; Pyo et al. 2003, 2006).

In this paper, we report the results of optical longslit spectroscopy on HH 158 and HH 702 outflows. We investigate the electron density, electron temperature, and ionization fraction from close vicinity of the central source to the knot at $14^{\prime \prime}$ of HH 158 jet and HH 702 at $650^{\prime \prime}$ from DG Tau. We note that this is the first time to obtain optical spectroscopy of the HH 702 outflow. We also summarize the proper motions of the knots in 
Table 1

Log of Observations.

\begin{tabular}{ccccccc}
\hline Object & Date (UT) & P.A. $\left(^{\circ}\right)$ & $\begin{array}{c}\text { Exposure } \\
\text { time }(\mathrm{s})\end{array}$ & $\begin{array}{c}\text { Slit } \\
\text { width }\left({ }^{\prime \prime}\right)\end{array}$ & $\begin{array}{c}\text { Spectral } \\
\text { resolution }(\lambda / \Delta \lambda)\end{array}$ & $\begin{array}{c}\text { Spectral } \\
\text { coverage }(\AA)\end{array}$ \\
\hline HH 158 & 2012 Nov. 15 & 223 & 1800 & 2.9 & 2070 & $5900-7050$ \\
HH 702 & 2012 Nov. 15 & 193 & 2800 & 2.9 & 2070 & $5900-7050$ \\
\hline
\end{tabular}

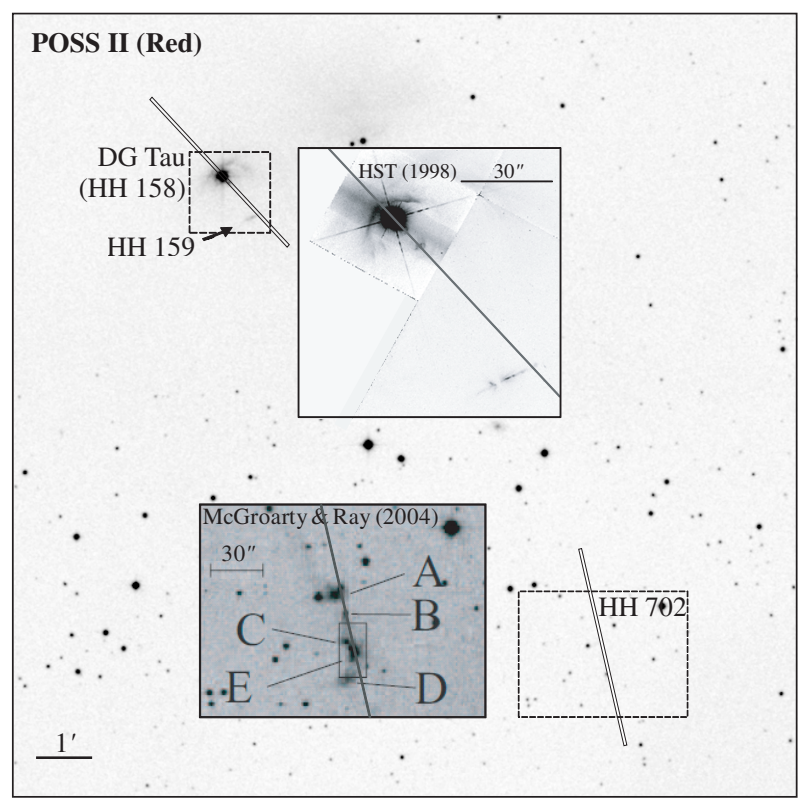

Figure 1. Slit positions on the object field. The center of the $2 . \prime 9(\mathrm{~W}) \times 3.6(\mathrm{~L})$ slit is set on HH 158 and HH 702 . The position angles are $223^{\circ}$ and $193^{\circ}$ for $\mathrm{HH} 158$ and $\mathrm{HH}$ 702 , respectively. The background image is from the Second Palomar Observatory Sky Survey (POSS II). The HST image of HH 158, 159 and $\mathrm{H} \alpha$ image of $\mathrm{HH} 702$ from McGroarty \& Ray (2004) are inserted as insets. Knot A - E of $\mathrm{HH} 702$ are marked.

the jet with the positions during the last 30 years. In Section 2, we describe the observation and data reduction. In Section 3, we show the line intensity and ratios in the position-velocity map. Sections 4 and 5 are the discussion and summary, respectively.

\section{ObSERVATion AND DAta REDUCtion}

The observation was conducted on November 15, 2012 (UT) at BOAO (Bohyunsan Optical Astronomy Observatory) with an optical long-slit spectrograph installed on the Cassegrain focus of a 1.8-m telescope. The wavelength coverage was $5900-7050 \AA$ with 1200 $\mathrm{gmm}^{-1}$ grism, which was chosen to achieve the emission lines of $\mathrm{H} \alpha$, [S II] $\lambda \lambda 6716,6731$, [N II] $\lambda \lambda 6548,6584$, [O I] $\lambda \lambda 6300,6363$. The slit length and width were 3.6 arc-minute and 2.9 arc-second, respectively. The resultant spectral resolution was $\sim 2070\left(150 \mathrm{~km} \mathrm{~s}^{-1}\right)$ with the dispersion of $0.41 \AA_{\text {Apixel }}^{-1}$. The CCD camera size is $4 \mathrm{k} \times 4 \mathrm{k}$ and the pixel scale along the slit is $0^{\prime \prime} 45$ pixel $^{-1}$. Exposures on comparison lines were taken with the FeNeArHe lamp before and after the

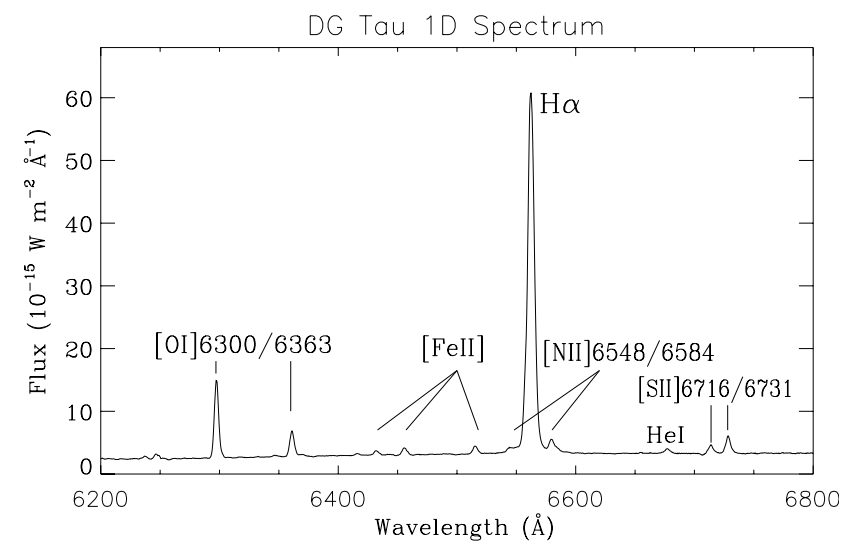

Figure 2. Integrated $1 \mathrm{D}$ spectrum of the central region of DG Tau.

exposure of each object, for wavelength calibration.

Table 1 shows the observation log of HH 158 and HH 702. Figure 1 shows the slit positions. The position angle (PA) of the $\mathrm{HH} 158$ region including the central star is determined to be $223^{\circ}$ (McGroarty \& Ray 2004) after verification through an HST image. In the case of $\mathrm{HH} 702$ located at $\sim 11^{\prime}$ from the star, a PA of $193^{\circ}$ was selected to obtain the emissions as much as possible. The exposure times were $1800 \mathrm{~s}$ and $2800 \mathrm{~s}$ for $\mathrm{HH} 158$ and $\mathrm{HH}$ 702, respectively. HR 1544 (A1 type) was observed for telluric line correction and flux calibration.

We reduced the data with standard IRAF (Image Reduction and Analysis Facility) packages. Bias was subtracted from every frame. The flat-fielding was carried out using a normalized flat made by the APFLATTEN task. Bad pixels and cosmic-ray events were corrected. The spectra were extracted using the APALL task. The wavelength calibration and distortion correction were accomplished by the IDENTIFY, REIDENTIFY, FITCOORDS, TRANSFORM tasks. There were 1 and 2 pixel shifts between the comparison frames before and after the exposure for $\mathrm{HH} 158$ and $\mathrm{HH}$ 702, respectively. Since the instrument is mounted on the Cassegrain focus, the instrumental displacement due to changes of the telescope elevation angle cause those shifts. We used the average of two lamp images to achieve a more accurate spectral calibration. There were also wavelength shifts between object frames. We corrected these shifts with the $\mathrm{OH}$ airglow lines in the object frames. After that, the sky emission lines were subtracted using the BACKGROUND task. The wavelength sensitivity correction and flux calibration were done with the standard star, HR 1544 (A1, V=4.35, $\left.\mathrm{T}_{\text {eff }}=9150 \mathrm{~K}\right)$. 


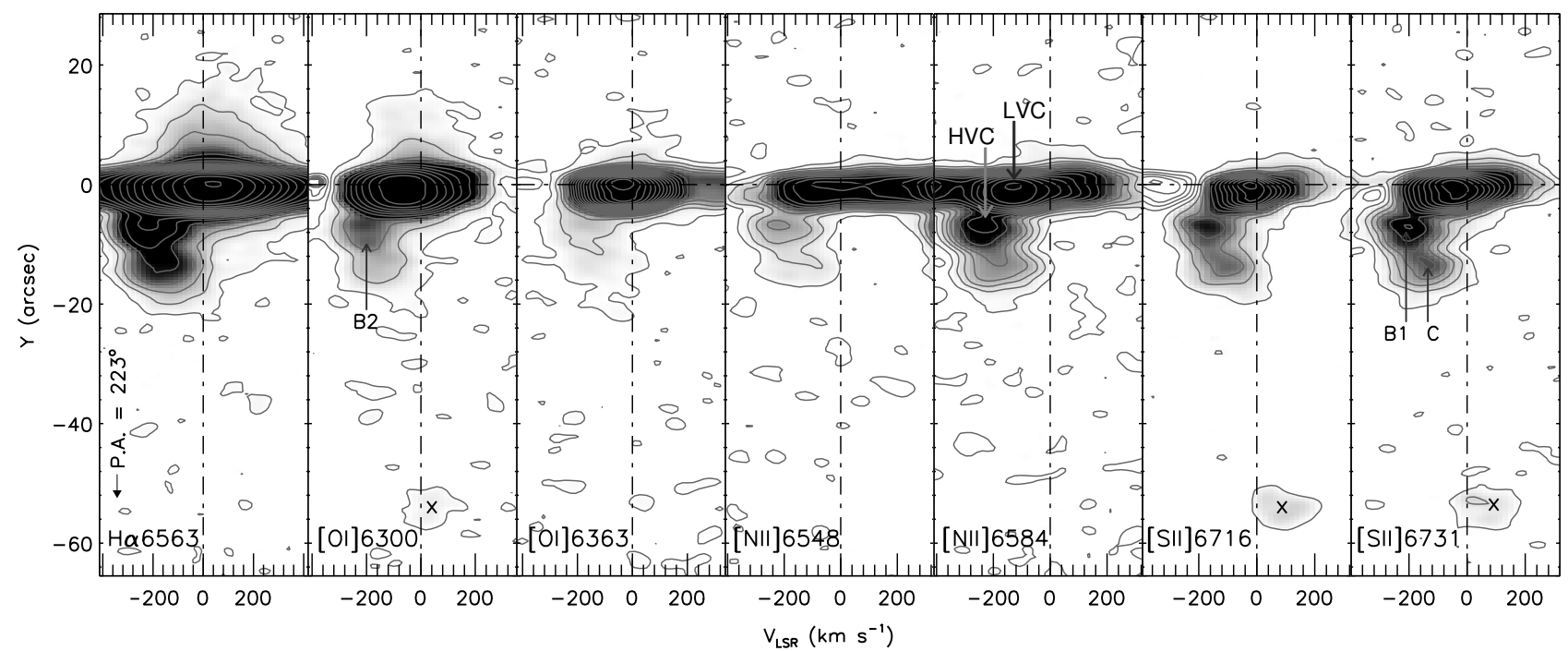

Figure 3. Continuum-subtracted PVDs of the emission lines of HH 158. Contours are drawn from 0.001 (3 $\sigma)$ to the peak levels of $5.2(\mathrm{H} \alpha), 0.98([\mathrm{O} \mathrm{I}] \quad \lambda 6300), 0.33$ ([O I $] \lambda 6363), 0.17$ ([N $\mathrm{II}] \lambda 6548$ and $\lambda 6584), 0.10$ ([S II $] \lambda 6716)$ and 0.22 $([\mathrm{S}$ II $] \lambda 6731) \times 10^{-15} \mathrm{~W} \mathrm{~m}^{-2} \AA^{-1}$ with an equal interval in a logarithmic scale. The position angle $\left(223^{\circ}\right)$ of the slit is marked in the left panel. Northeast is up and southwest is down in the diagram. The original spectrum has Gaussian Smoothed with a $\sigma$ value of $1.5 \times 1.5$. Knots B1, B2 (Lavalley et al. 1997), and C (Eislöffel \& Mundt 1998) are identified at $\mathrm{Y} \sim-6^{\prime \prime}$ and $-14^{\prime \prime}$. The faint emissions at $\mathrm{Y}=-54^{\prime \prime}$ in [O I] $\lambda 6300$, [S II] $\lambda 6716$ and [S II] $\lambda 6731$ are parts of the HH 159 outflow ejected from DG Tau B. These are labeled with the ' $X$ ' symbols in the figure.

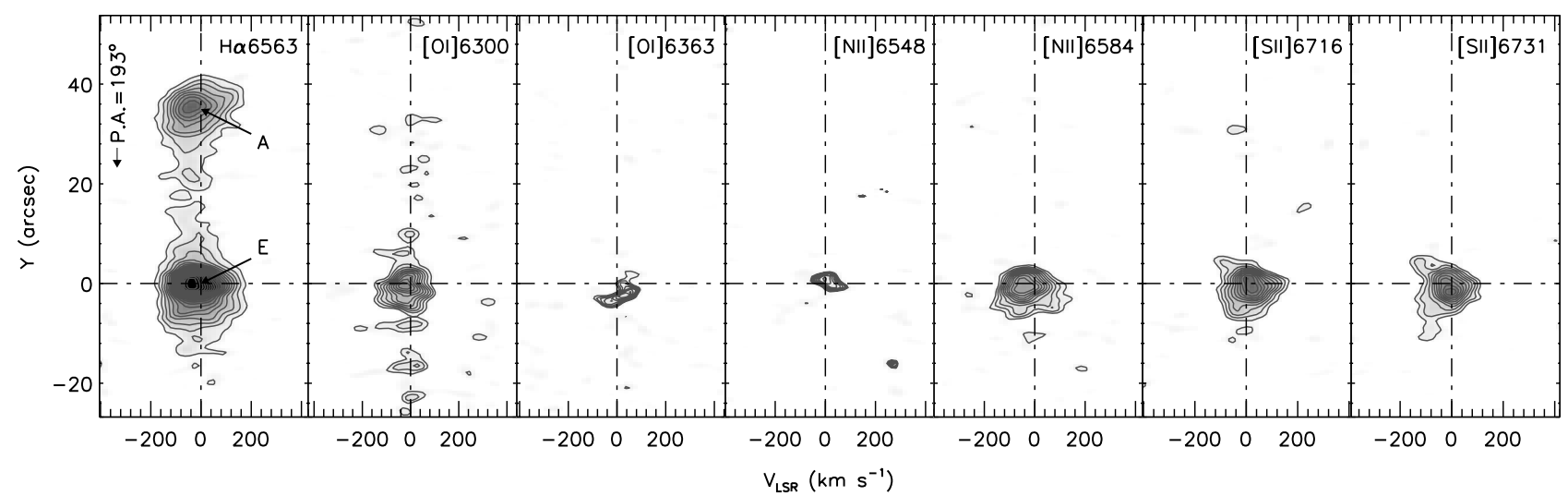

Figure 4. PVDs of the emission lines of HH 702. Contours are drawn from 0.05 to the peak levels of $1.5(\mathrm{H} \alpha)$, 0.45 $([\mathrm{O} \mathrm{I}] \lambda 6300), 0.12([\mathrm{O} \mathrm{I}] \lambda 6363), 0.11$ ([N II $] \lambda 6548), 0.38$ ([N II] $\lambda 6584), 0.60$ ([S II] $\lambda 6716)$, and 0.58 ([S II $] \lambda 6731)$ in $10^{-17}$ $\mathrm{W} \mathrm{m} \mathrm{m}^{-2} \AA^{-1}$ with an equal interval in a logarithmic scale. The position angle $\left(193^{\circ}\right)$ of the slit is marked in the left panel. Northeast is up and southwest is down in the diagram. The knots A and E of McGroarty \& Ray (2004) are identified. 

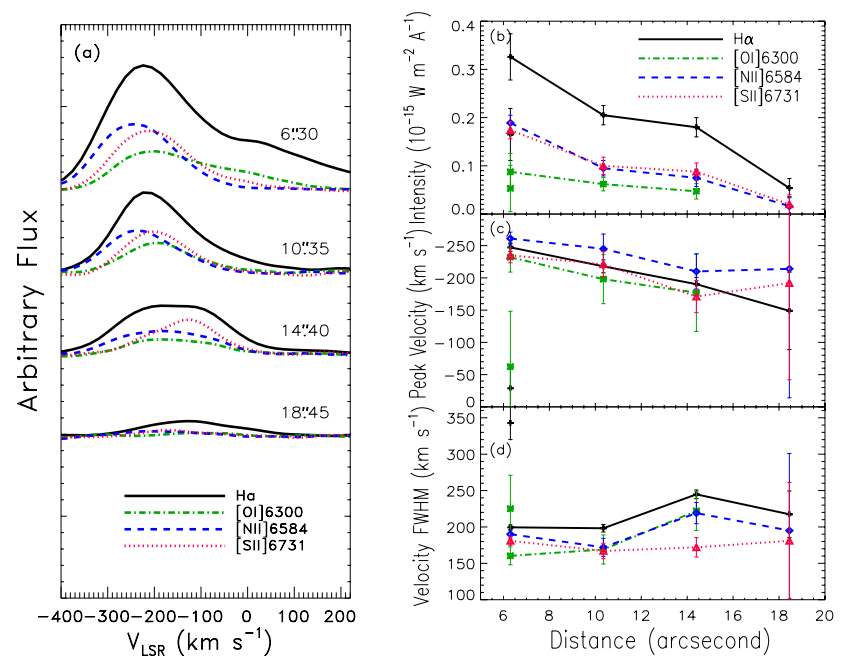

Figure 5. (a) Variation of the line profile, $(b)$ peak intensity, (c) peak velocity, and $(d)$ velocity width (FWHM) of the each emission line of $\mathrm{HH} 158$ with the distance from DG Tau.

We removed a deep $\mathrm{H} \alpha$ absorption line in the spectrum of the standard star using the SPLOT task before applying the wavelength sensitivity correction.

\section{Results}

\subsection{Kinematics}

Figure 2 shows the 1D spectrum extracted from the central source region within \pm 5 ". 4 from DG Tau. In the wavelength range of $6200-6800 \AA$, the $\mathrm{H} \alpha \lambda 6563$, $\left[\begin{array}{ll}\mathrm{O} & \mathrm{I}\end{array}\right] \lambda \lambda 6300,6363, \quad[\mathrm{~N} \quad \mathrm{II}] \quad \lambda \lambda 6548,6584$, and [S II] $\lambda \lambda 6716,6731$ emissions were detected. The [Fe II] emissions at $\lambda 6433, \lambda 6456, \lambda 6516 \AA$ and the atomic Helium line at $\lambda 6678 \AA$ are also identified. the $\mathrm{H} \alpha$ emission has the highest intensity peak level of 6 $\times 10^{-14} \mathrm{~W} \mathrm{~m}^{-2} \AA^{-1}$, which is $\sim 30$ times higher than that of the stellar continuum. The wing of the strong $\mathrm{H} \alpha$ line affects the line profiles of two [N II] lines.

Figures 3 and 4 show the position-velocity diagrams (PVDs) of HH 158 and HH 702. In Figure 3, the stellar continuum was subtracted. The position at $\mathrm{Y}=0^{\prime \prime}$ indicates DG Tau and the peak of knot $\mathrm{E}$ in Figures 3 and 4, respectively. HH 702 is located at $\sim 11^{\prime}(=$ $0.45 \mathrm{pc}$ at $140 \mathrm{pc}$ distance) southwest from DG Tau, as shown in Figure 1. The Gaussian smoothing was applied in both figures with sigma of $1.5 \times 1.5$ pixels.

In Figure 3, we identify the knots B1, B2 (Lavalley et al. 1997) and $C$ (Eislöffel \& Mundt 1998) at the distance of $66^{\prime \prime} 74,8^{\prime \prime} .5$ and $\sim 14^{\prime \prime}$ from the star. The two velocity components appear in all emission lines in Figure 3 . The low velocity component (LVC) is located within $\pm 5^{\prime \prime}$. The peak velocity of the LVCs varies from $\sim-80$ to $-20 \mathrm{~km} \mathrm{~s}^{-1}$. The high velocity component (HVC) is relevant at $|\mathrm{Y}|>6^{\prime \prime}$. Figure $5 c$ shows that the HVC is getting slower with increasing distance, e.g., -270 $\mathrm{km} \mathrm{s}^{-1}$ at $6^{\prime \prime} 3$ (knot B1) and $-150 \mathrm{~km} \mathrm{~s}^{-1}$ at $18^{\prime \prime} 45$. The real jet velocity is 1.18 times higher than the radial velocity considering the inclination angle of $32^{\circ}$ with re-

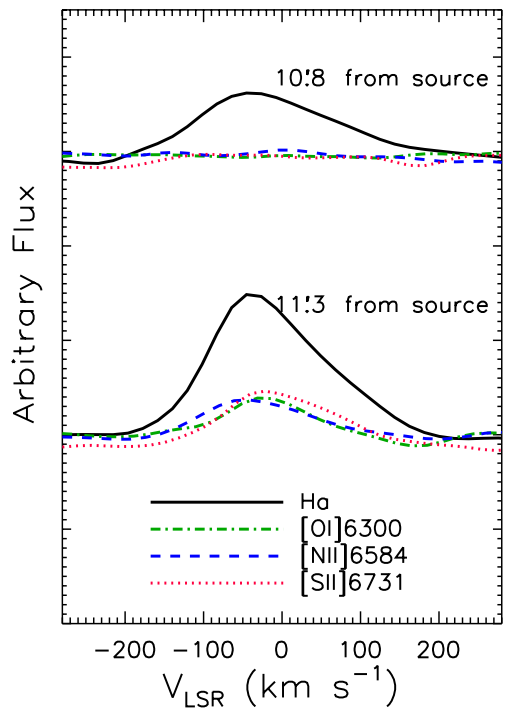

Figure 6. Line profiles at knots A (upper) and $\mathrm{E}$ (lower) of HH 702.

spect to the line of sight (Eislöffel \& Mundt 1998), so the radial velocity of $-270 \mathrm{~km} \mathrm{~s}^{-1}$ corresponds to the jet velocity of $-320 \mathrm{~km} \mathrm{~s}^{-1}$. Additionally, it is noticeable that the emission lines are showing different spatial extents. In Figure 3, all emissions except [N II] $\lambda 6548$ show extensions to $\mathrm{Y} \sim-20^{\prime \prime}$ at $3 \sigma$ contour levels. Knot B1 is relevant in all emissions except [O I] $\lambda 6363$. Knot $\mathrm{C}$ is remarkable in $[\mathrm{S} \mathrm{II}] \lambda 6731$ and appears as plateaus in $\mathrm{H} \alpha$, [O I] $\lambda 6300$, [N $\mathrm{II}] \lambda 6584$, and $[\mathrm{S}$ II] $\lambda 6716$. Its intensity in [N II] $\lambda 6584$ is almost twice than that of $[\mathrm{O} \mathrm{I}] \lambda 6300$.

The faint emissions at a distance of $54^{\prime \prime}$ from DG Tau, detected at $[\mathrm{O} \mathrm{I}] \lambda 6300,[\mathrm{~S} \mathrm{II}] \lambda 6716$ and $[\mathrm{S} \mathrm{II}] \lambda 6731$ may not come from $\mathrm{HH} 158$ but from a part of $\mathrm{HH} 159$ (see Figure 1), which is ejected from DG Tau B. These features are labeled with ' $x$ ' symbols.

In Figure 4, we identified the knots $\mathrm{A}$ and $\mathrm{E}$ of McGroarty \& Ray (2004). The knot A of HH 702 is bright only in $\mathrm{H} \alpha$ emission, while it is seen in the [S II] images of McGroarty \& Ray (2004) and Sun et al. (2003). Knot $\mathrm{E}$ is detected in all emission lines.

Figure 5 shows the variations of the line profile, peak intensity, peak velocity, and velocity width (FWHM) of each emission line with the distance from the star for $\mathrm{HH}$ 158. The data are sampled at 6.'30, 10."35, 14.' 40 , and $18^{\prime \prime} .45$ along the blueshifted jet. The plot starts from $66^{\prime \prime} 30$ where the knot B1 is located. The [O I] $\lambda 6300$ emission at $18^{\prime \prime} 45$ could not be measured due to faintness. In Figures $5 b$ and $5 c$, the peak intensities and velocities of all the emission lines decrease with distance from the source. The uncertainty in the peak velocity measurement of [N II] and [S II] at 18 ". 45 is $\pm 170 \mathrm{~km} \mathrm{~s}^{-1}$. It is large, due to low a signal-to-noise ratio $(\mathrm{S} / \mathrm{N}<2)$. Even though there are small increases in velocity (Figure $5 c$ ), those are not significant due to a large uncertainty. The decreasing rate in the velocity of $\mathrm{H} \alpha$ is $\left|\Delta v_{\text {peak }}\right| \sim 7 \mathrm{~km} /{ }^{\prime \prime}$.

In Figure $5 d$, the range of velocity widths (FWHM) 

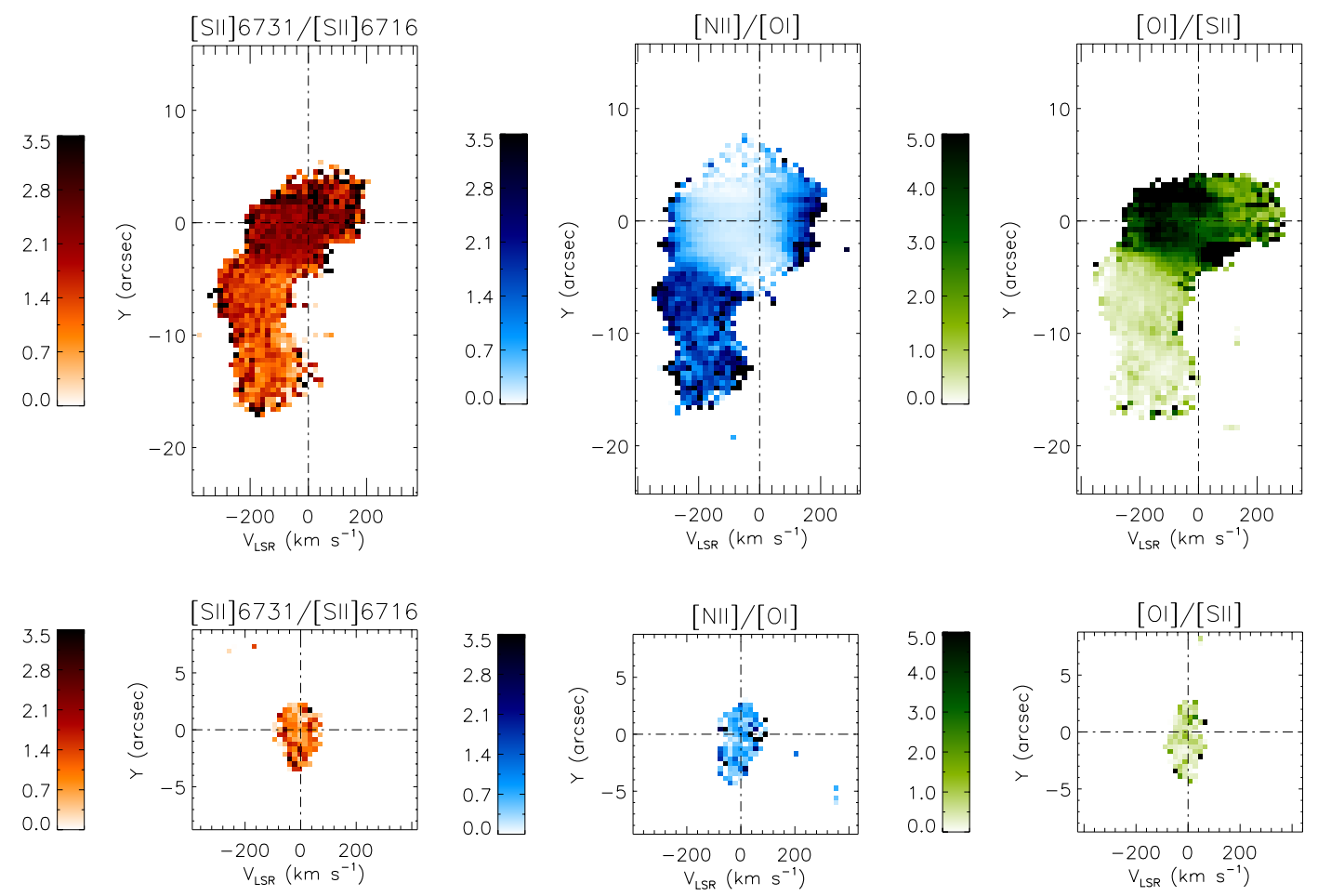

Figure 7. Ratios of forbidden emission lines: (top) HH 158 and (bottom) knot E of HH 702. (Left) [S II] $\lambda 6731 / \lambda 6716$, (center) [N II] $\lambda 6548+\lambda 6584 /[\mathrm{O}$ I] $\lambda 6300+\lambda 6363$, and (right) [O I] $\lambda 6300+\lambda 6363 /[\mathrm{S} \mathrm{II}] \lambda 6716+\lambda 6731$.

varies from 150 to $350 \mathrm{~km} \mathrm{~s}^{-1}$, which is marginally resolved considering the velocity resolution of $150 \mathrm{~km} \mathrm{~s}^{-1}$ in our observation. The low velocity component shows a wider width $\left(\sim 340 \mathrm{~km} \mathrm{~s}^{-1}\right.$ in $\left.\mathrm{H} \alpha\right)$ than those estimated for high velocity components $\left(\sim 160-230 \mathrm{~km} \mathrm{~s}^{-1}\right)$, compatible with results obtained in previous studies (e.g., Hirth et al. 1997; Pyo et al. 2003). Pyo et al. (2002) interpreted the wider velocity width of LVC as follows: if we assume that the outflow has the shape of a diverging wind, the velocity width represents the difference in radial velocities between the nearest and farthest streamlines from us. Thus, the wider velocity width means a wider opening angle.

Figure 6 shows the variations of the line profile along the distance of $\mathrm{HH} 702$. The peak velocities of two knots are $\sim-80 \mathrm{~km} \mathrm{~s}^{-1}$ in the $\mathrm{H} \alpha$ emission.

\subsection{Line Ratios}

The diagnostics by the ratios of forbidden emission lines is a very effective tool to study jet properties (Hartigan et al. 1994; Bacciotti et al. 1995). In Figure 7, we show the flux ratios of the forbidden emisson lines of $\mathrm{HH}$ 158 and knot $\mathrm{E}$ of HH 702 in PVDs : [S II] $\lambda 6731$ / $\lambda 6716,[\mathrm{~N}$ II] $\lambda 6548+\lambda 6584 /[\mathrm{O}$ I] $\lambda 6300+\lambda 6363$, and $\left[\begin{array}{ll}\mathrm{O} & \mathrm{I}\end{array} \lambda 6300+\lambda 6363 /[\mathrm{S}\right.$ II $] \lambda 6716+\lambda 6731$. The ratio of the [S II] doublet can be used to estimate the electron density (Osterbrock 1989), which is larger than 2 within $\pm 5^{\prime \prime}$ and decreases with the distance. The $\left[\begin{array}{ll}\mathrm{N} & \mathrm{II}\end{array}\right] /\left[\begin{array}{ll}\mathrm{O} & \mathrm{I}\end{array}\right]$ ratio, which is proportional to the ionization fraction of hydrogen, is less than 0.5 at the center and increases at distances further than $\sim 5^{\prime \prime}$. The $[\mathrm{O} \mathrm{I}] /\left[\begin{array}{ll}\mathrm{S} & \mathrm{II}\end{array}\right]$ ratio shows a similar trend with the

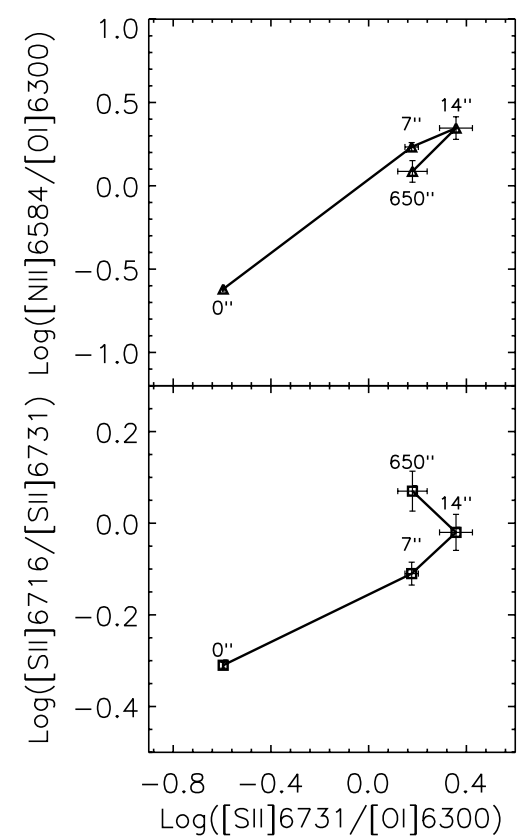

Figure 8. Line ratios estimated from HH 158 and $\mathrm{HH} 702$, for four distances: $d=0^{\prime \prime}, 7^{\prime \prime}, 14^{\prime \prime}, 650^{\prime \prime}$.

[S II] doublet, large near the center and less than 1.0 further than $5^{\prime \prime}$. This ratio is related to the electron temperature. The line ratios of HH 702 are comparable with those obtained from $\mathrm{HH} 158$ at $\sim 14^{\prime \prime}$ from the source. In Figure 8, we show the relations between three ratios, [S II] $\lambda 6731 /[\mathrm{O}$ I] $\lambda 6300,[\mathrm{~N}$ II] $\lambda 6584 /$ [O I] $\lambda 6300$, and $[\mathrm{S}$ II] $\lambda 6716 / \lambda 6731$. In the figure, the 
Table 2

Locations of the knots with respect to the source position during the last 30 years.

\begin{tabular}{lcccc}
\hline & \multicolumn{3}{c}{ Distance $\left(^{\prime \prime}\right)$} & \\
\cline { 2 - 4 } Observation date & B1 $^{\mathrm{a}}$ & $\mathrm{B}^{\mathrm{a}}$ & $\mathrm{C}^{\mathrm{b}}$ & Reference \\
\hline 1984 Oct. 9 & - & - & 8.5 & Mundt et al. (1987) ${ }^{\mathrm{c}}$ \\
1986 Dec. & - & - & 8.7 & Eislöffel \& Mundt (1998) \\
1992 Oct. & 2.25 & 3.25 & - & Solf \& Böhm (1993) \\
1994 Nov. 3 & 2.7 & 3.9 & - & Lavalley et al. (1997) \\
1997 Jan. & 3.3 & - & - & Dougados et al. (2000) \\
1998 Jan. 23-26 & 3.6 & - & - & Lavalley-Fouquet et al. (2000) \\
1998 Feb. 5 & - & - & 10.65 & HST \\
1999 Jan. 14 & 3.8 & - & - & Maurri et al. (2014) \\
2002 Sep. 17 & 4.6 & - & 12 & Whelan et al. (2004) \\
2012 Nov. 15 & 6.74 & 8.75 & 13.7 & This work \\
2014 Nov. 21 & 7.65 & - & 13.5 & This work \\
Proper motion $\left(^{\prime \prime}{ }^{-1}\right)$ & 0.230 & 0.272 & 0.180 & - \\
Ejection date & 1981 & 1980 & 1939 & - \\
\hline
\end{tabular}

${ }^{a}$ knot name from Lavalley et al. (1997).

b knot name from Eislöffel \& Mundt (1998).

c position of knot C measured from Figure 9 of Mundt et al. (1987).

d data taken from the HST archive, image of WFPC2 with the F675WF filter (proposal ID. 6855).

four points represent the values at distances $d=0^{\prime \prime}, 7^{\prime \prime}$, $14^{\prime \prime}, 650^{\prime \prime}$ from the source. The distance of $650^{\prime \prime}$ is the location of the knot $\mathrm{E}$ of $\mathrm{HH} 702$. The ratios increase with distance, as in a previous study (Lavalley-Fouquet et al. 2000, Figure 3a-b).

\section{Discussion}

\subsection{Velocity Variation}

In Figure 3, the HVC is getting slower with distance. The velocity decreasing rate derived in Section 3 is $\sim 7$ $\mathrm{km} /{ }^{\prime \prime}$. If the jet is continuously slowing, then it should stop at $35^{\prime \prime}$ from DG Tau. However, the knots of HH 702 located at $650^{\prime \prime}$ show a radial velocity of $\sim-80$ $\mathrm{km} \mathrm{s}^{-1}$.

Radial velocity $\left(v_{\text {rad }}\right)$ measurements of knot $\mathrm{C}$ in $\mathrm{HH} 158$ were reported in several previous studies. The knot was observed at 8..5 (Mundt et al. 1987) and 8..7 (Eislöffel \& Mundt 1998) from the source in 1984 and 1987, respectively. We converted the heliocentric velocities to LSR velocities for the comparison with our data. In Mundt et al. (1987), $v_{\text {rad }}$ were $-136 \mathrm{~km} \mathrm{~s}^{-1}$ in $\mathrm{H} \alpha$ and $-123 \mathrm{~km} \mathrm{~s}^{-1}$ in [S II] $\lambda 6731$. In Eislöffel \& Mundt (1998), [S II] is $\sim-146 \mathrm{~km} \mathrm{~s}^{-1}$. In 2002 (Whelan et al. 2004), [S II] $\lambda 6731$ and [O I] $\lambda 6300$ show $v_{\text {rad }}$ of $\sim-150$ and $-200 \mathrm{~km} \mathrm{~s}^{-1}$, respectively. For the three emission lines mentioned above $(\mathrm{H} \alpha,[\mathrm{S} \mathrm{II}],[\mathrm{O} \mathrm{I}])$, our data show $v_{\text {rad }}$ of $-190,-128$, and $-180 \mathrm{~km} \mathrm{~s}^{-1}$, respectively. Since different emission lines originate from different excitation conditions (i.e., temperature, density, etc.), they represent various regions in the outflow and show peaks in different velocities. The temporal variation of the velocity indicates that the velocity of knot is variable not only along the distance but also in time. This may imply that the ejected knot has been decelerated when it passes through the ambient medium. The velocity change of the knot could also occur due to the

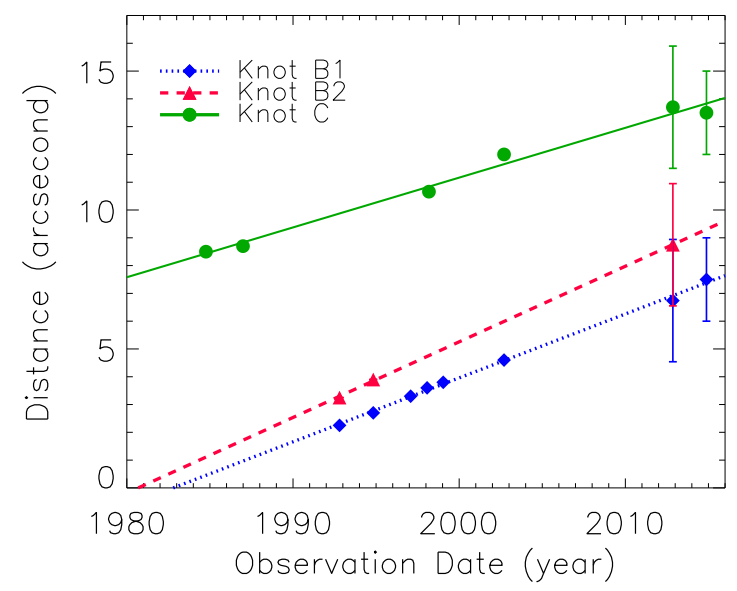

Figure 9. Positional variations of three knots. For knot B1, the data points are expanded from those in Pyo et al. (2003).

shock caused by the interaction between the faster gas stream behind and the slower front knot.

\subsection{Proper Motion}

In the emission lines of $\mathrm{HH} 158$, we have distinguished three knots at $6{ }^{\prime \prime} .74$ and 8 .' 75 , and $13^{\prime \prime} .7$ from the source in our data taken on November 15, 2012. We identified these knots as B1 and B2 of Lavalley et al. (1997), and knot C of Eislöffel \& Mundt (1998). Recently, we could measure the knot positions from our additional data taken on November 21, 2014. For knot B1 and C, it was measured to 7 ". $^{\prime} 65$ and 13 .' $^{\prime}$, respectively. We note that knot B2 is not resolved in our spectra in 2014. The distance of knot $\mathrm{C}$ from the source is $0 . \prime 2$ smaller in 2014 than that in 2012. The seeing size is $>2^{\prime \prime}$, so it may be due to the uncertainty of the measurement. 


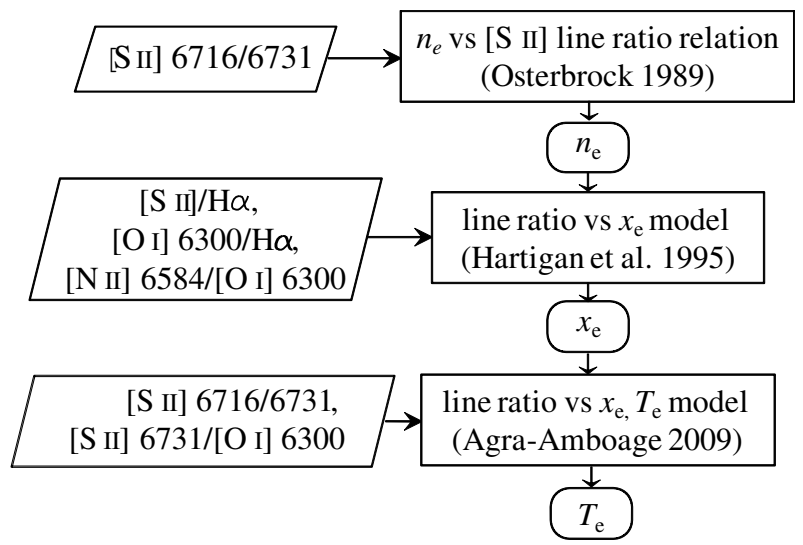

Figure 10. Flow chart showing how we derive the electron density $\left(n_{e}\right)$, ionization fraction $\left(x_{e}\right)$, and electron temperature $\left(T_{e}\right)$.

In Table 2, we list the locations of the knots during the last $\sim 30$ years including our work. Basically, the positions of knot B1 are extended from Table 1 of Pyo et al. (2003). In the case of knot $\mathrm{C}$, the first detection was reported in 1984 (Mundt et al. 1987) and it was seen at $12^{\prime \prime}$ in long-slit spectroscopy of Whelan et al. (2004) whose data were taken in 2002. In addition to these data points, we found an Hubble Space Telescope (HST) archival image obtained in 1998. The central peak in intensity contour of the knot in the HST image is located about $10^{\prime \prime} 65$ from DG Tau.

Figure 9 shows the temporal variation of the distance for the knots B1, B2, and C. We estimated the proper motion by linear regression: 0 ' $230 \pm 0$.'006 $\mathrm{yr}^{-1}$ for $\mathrm{B} 1,00^{\prime \prime} 272 \pm 0$. .'002 $\mathrm{yr}^{-1}$ for B2, $0.180 \pm 0$. .'008 $\mathrm{yr}^{-1}$ for C (see Table 2). For knot B1, the estimated value is smaller than those obtained from previous studies, e.g., 0'.272 in Pyo et al. (2003). Due to the large spatial resolution of our observation, the emission from knot B1 could be combined with that from knot A1 which has identified from previous studies (Bacciotti et al. 2000; Dougados et al. 2000). The smaller proper motion value of knot B1 may be estimated because knot A1 is located $2 . .5$ closer (Maurri et al. 2014) to the star than knot B1. From the radial and the tangential velocities of each knots, the inclination angles $(i)$ of $\mathrm{B} 1, \mathrm{~B} 2$, and $\mathrm{C}$ are calculated as $32.5 \pm 0.7,37^{\circ} .0 \pm 0.2,32^{\circ} .3 \pm 1.1$ with respect to the line of sight.

For HH 702, McGroarty et al. (2007) estimated the tangential velocities of knots $\mathrm{A}$ and $\mathrm{E}$ as $129 \pm(10-$ $28)$ and $186 \pm(10-28) \mathrm{km} \mathrm{s}^{-1}$ in the $\mathrm{H} \alpha$ emission, which correspond to $0^{\prime \prime} .19 \pm 0$. . 03 and $0^{\prime \prime} .28 \pm 0$. . $03 \mathrm{yr}^{-1}$ in proper motion, respectively. From the radial velocity of $\sim-80 \mathrm{~km} \mathrm{~s}^{-1}$ and the tangential velocities, the $i$ of knot $\mathrm{A}$ and $\mathrm{E}$ are estimated as $\sim 58.2 \pm 1.1$ and $\sim 66.7$ \pm 1 . 1 with respect to the line of sight. The $i$ of $\mathrm{HH}$ 702 are $\sim 21-35^{\circ}$ different from those of $\mathrm{HH} 158$. We cannot avoid the possibility that the two $\mathrm{HH}$ objects were ejected from different sources although McGroarty et al. (2007) postulated that DG Tau seems the most probable driving source of HH 702 based on the proper motion direction. The different direction or the curved shape of a jet may indicate the precession of the jet (Raga et al. 2001).

\subsection{Line Ratios and Physical Parameters}

Maurri et al. (2014) shows the line ratios and physical parameters within $5^{\prime \prime}$ of DG Tau. Their [S II] $\lambda 6731 / \lambda 6716$ ratio is similar to our value. Knots B0 and B1 at 3.' 3 and 3". 8 in Maurri et al. (2014) correspond to $\mathrm{B} 1$ at 6.77 in our data, after considering the proper motion. For this knot, the [S II] doublet ratio in our data is slightly smaller than their estimation. For the $[\mathrm{N} \mathrm{II}] /[\mathrm{O} \mathrm{I}]$ ratio, the value in Maurri et al. (2014) is $0.01-2.5$, in a similar range as our data.

In Figure 10, the flow chart showing how we estimate the electron density $\left(n_{e}\right)$, ionization fraction $\left(x_{e}\right)$, and electron temperature $\left(T_{e}\right)$ is displayed. Figure 11 shows the derived physical parameters for $\mathrm{HH} 158$ and $\mathrm{HH}$ 702. The estimated properties of each knot show good agreement with the planar shock model (Hartigan et al. 1994; Lavalley-Fouquet et al. 2000). For the variation of the jet with the distance (i.e., variation with time), the model that treats the time variability of the jet (Raga et al. 1990; Raga \& Kofman 1992) could explain our result with the internal shock caused by a variable (or periodic) jet ejection.

In our data, it is hard to distinguish the two velocity components within $\pm 5^{\prime \prime}$ from DG Tau due to the limitations in spatial and velocity resolution. The LVC is usually dominant close to the source, while HVC is extended further as shown in Figure 3. With this point of view, it is noticeable that the $x_{e}$ of HVC at $|\mathrm{Y}|>$ $4^{\prime \prime}$ is higher than that of the LVC at $|\mathrm{Y}|<4^{\prime \prime}$ but $n_{e}$ is lower in HVC in Figure 11. One the other hand, previous studies (Lavalley-Fouquet et al. 2000; Maurri et al. 2014) show that the $n_{e}$ and $x_{e}$ are larger in their high and medium velocity interval than those in low velocity. Hamann (1994) also showed that the two velocity components of 20 TTS and 12 HAeBe stars are different in $T_{e}$ and $n_{e}$. These results indicate that the two velocity components have different physical conditions. They are supposed to originate from different parts of star-disk systems or have different launching mechanisms (Kwan \& Tademaru 1995; Pyo et al. 2003, 2006).

\subsubsection{Electron density $\left(n_{e}\right)$}

We calculated the electron density using the typical method of [S II] $\lambda 6716 / \lambda 6731$ doublet ratio (Osterbrock 1989). This ratio is known to be insensitive to temperature variation, so we assumed the electron temperature as $\sim 10,000 \mathrm{~K}$ to estimate the electron density $\left(n_{e}\right)$. In the bottom panel of Figure 12, $n_{e}$ is shown at four different distance positions. At the proximity of the star, $n_{e}$ is $\sim 10^{4} \mathrm{~cm}^{-3}$ and decreases to $\sim 500 \mathrm{~cm}^{-3}$ at $14^{\prime \prime}$ from the source. In HH 702 at $650^{\prime \prime}, n_{e}$ is estimated to be $\sim 200 \mathrm{~cm}^{-3}$. 

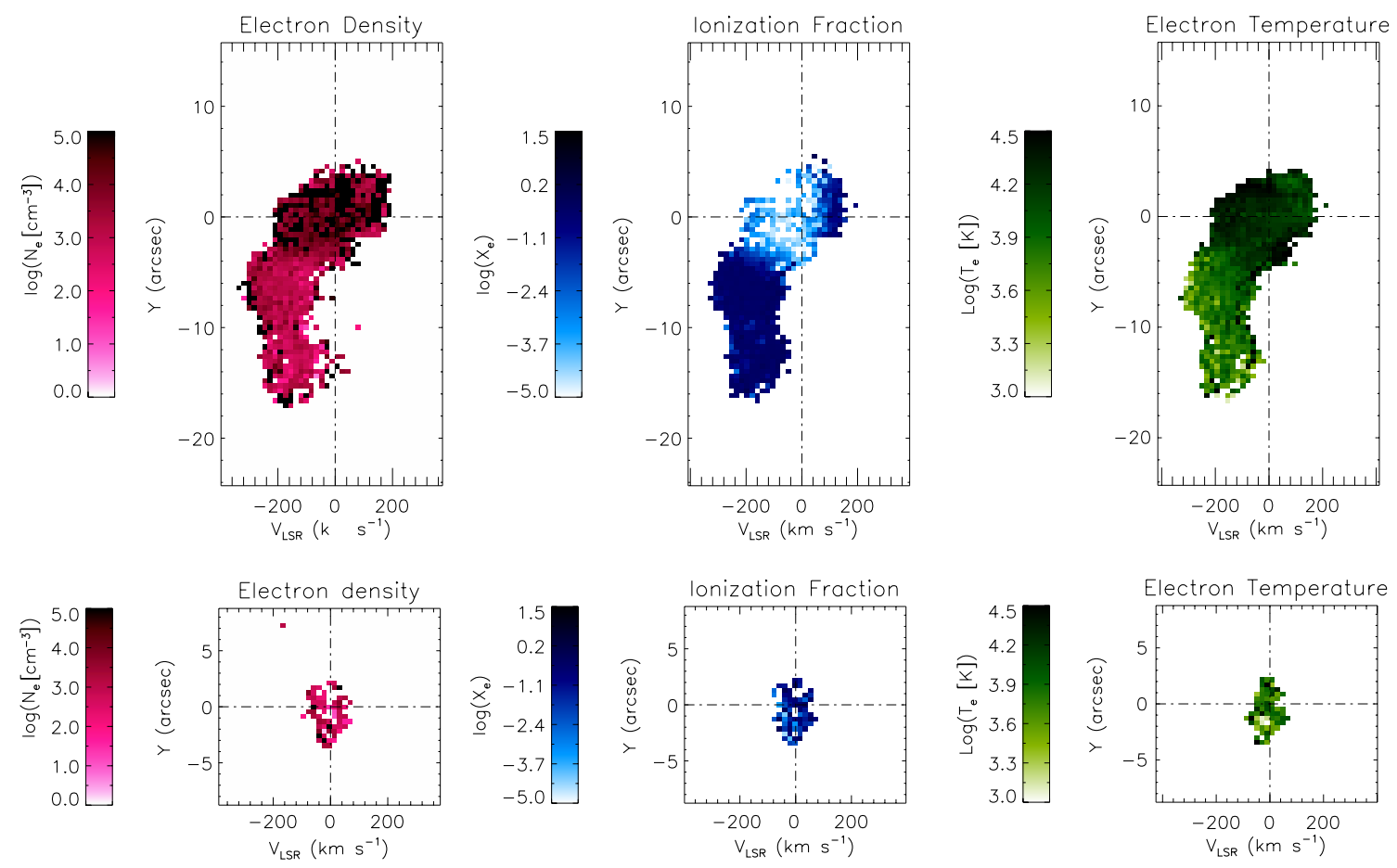

Figure 11. (Left) Electron density $\left(n_{e}\right)$, (center) ionization fraction $\left(x_{e}\right)$, and (right) electron temperature $\left(T_{e}\right)$ of HH 158 and $\mathrm{HH} 702$.

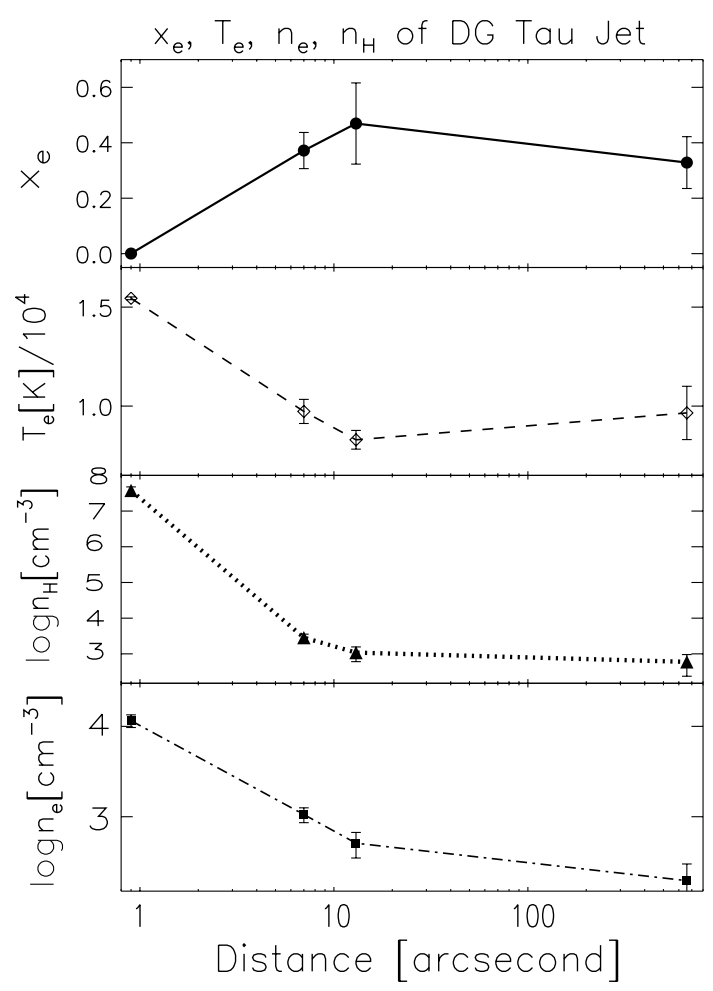

Figure 12. (From top to bottom) Ionization fraction $\left(x_{e}\right)$, electron temperature $\left(T_{e}\right)$, total hydrogen density $\left(n_{H}\right)$, and electron density $\left(n_{e}\right)$ of DG Tau jet as a function of the distance from the source.

\subsubsection{Ionization fraction $\left(x_{e}\right)$}

We estimated the ionization fraction from the model grid of Hartigan et al. (1994), which shows the relations between the ionization fraction and the line ratio of $\left[\begin{array}{ll}\mathrm{O} & \mathrm{I}\end{array}\right] \lambda 6300 / \mathrm{H} \alpha,[\mathrm{S} \quad \mathrm{II}] \quad \lambda 6716+\lambda 6731 / \mathrm{H} \alpha$,

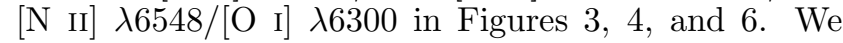
assumed the magnetic strength of $100 \mu \mathrm{G}$, close to the average value in the model. The middle panel of Figure 11 and the top panel of 12 show the ionization fraction obtained from the $[\mathrm{N} \mathrm{II}] /[\mathrm{O} \mathrm{I}]$ ratio. Figure 13 shows the $x_{e}$ values derived from three different ratios of [O I] $\lambda 6300 / \mathrm{H} \alpha$, [S II] $\lambda 6716+\lambda 6731 / \mathrm{H} \alpha$, [N II] $\lambda 6548 /\left[\mathrm{O}\right.$ I] $\lambda 6300$. The $x_{e}$ values deduced by $[\mathrm{O} \mathrm{I}] / \mathrm{H} \alpha$ and $[\mathrm{S} \mathrm{II}] / \mathrm{H} \alpha$ ratios are in the range of $0.02-$ 0.2 which are much different from the values deduced from $[\mathrm{N} \mathrm{II}] /[\mathrm{O}$ I]. Since the $\mathrm{H} \alpha$ emission flux comes not only from hydrogen recombination but also from collision (Bacciotti \& Eislöffel 1999; Hartigan 2003), the use of the $\mathrm{H} \alpha$ emission in the estimation of the ionization fraction may cause uncertainty. Thus, we used only the [N II]/[O I] ratio in the calculation of $x_{e}$.

In Figures 11 and 12, $x_{e}$ varies from $\sim 10^{-3}$ to $\sim$ 0.5 with distance from the source. According to Figure 16 of Hartigan et al. (1994) which shows the relation between ionization fraction and shock velocity, the shock velocity surpasses $100 \mathrm{~km} \mathrm{~s}^{-1}$ with $x_{e}$ of $\sim 0.5$ around knot $\mathrm{C}$, which has $\sim 190 \mathrm{~km} \mathrm{~s}^{-1}$ radial velocity. Lavalley-Fouquet et al. (2000) showed that $x_{e}$ was increasing from 0.02 to 0.6 in $\mathrm{HVC}$ and from 0.004 to 0.1 in LVC within $4^{\prime \prime}$ from the source. Recently, Maurri et al. (2014) reported similar results within $5^{\prime \prime}$ using HST/STIS data obtained in 1999. 


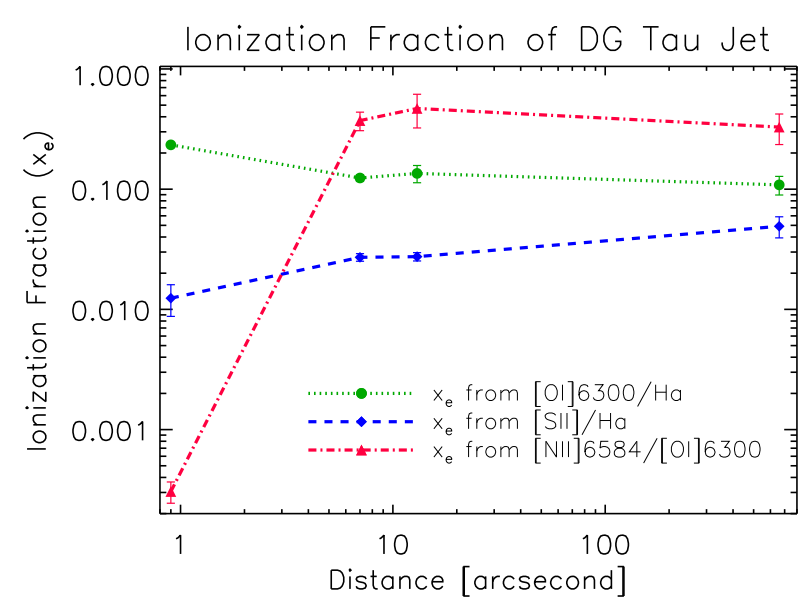

Figure 13. Ionization fraction $\left(x_{e}\right)$ derived from the line ra-

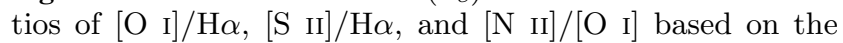
model of Hartigan et al. (1994).

For $\mathrm{HH} 702, x_{e}$ is greater than 0.2 , which is similar to the value at $14^{\prime \prime}$ from the source.

\subsubsection{Electron temperature $\left(T_{e}\right)$}

Electron temperature $\left(T_{e}\right)$ values are shown in Figures 11 and 12, and are estimated from the model grid of Figure 3.6 in Agra-Amboage (2009) which shows the relation between emission line ratios and electron density, temperature, and ionization fraction. Using the $x_{e}$ values obtained from 4.3.2, we have calculated the temperature from the model. $T_{e}$ is higher than 15,000 $\mathrm{K}$ in the central region and decreases to $\sim 5,000 \mathrm{~K}$ at $14^{\prime \prime}$. At HH 702, the temperature was estimated to be slightly higher than that measured at knot $\mathrm{C}$ of $\mathrm{HH}$ 158 .

\subsection{Mass-loss Rate}

The mass-loss rate of DG Tau was estimated in several previous studies. Hartigan et al. (1995) estimated the value of $3.2 \times 10^{-7} M_{\odot} \mathrm{yr}^{-1}$ from the [O I] $\lambda 6300$ emission line flux. In Lavalley-Fouquet et al. (2000), they have obtained the total value of $1.4 \times 10^{-8} M_{\odot} \mathrm{yr}^{-1}$ from the low, intermediate, and high velocity interval. The lower limit of mass-loss rate estimated by Beck et al. (2010) using the high-velocity $\operatorname{Br} \gamma$ emission was 1.2 $\times 10^{-8} M_{\odot} \mathrm{yr}^{-1}$. Agra-Amboage et al. (2011) has estimated a total mass-loss rate from $-300 \mathrm{~km} \mathrm{~s}^{-1}$ to $-50 \mathrm{~km} \mathrm{~s}^{-1}$ components of the [Fe II] emission to $3.3 \times$ $10^{-8} M_{\odot} \mathrm{yr}^{-1}$. Maurri et al. (2014) also has estimated a mass-loss rate in the range of $10^{-8}$ to $10^{-9} M_{\odot} \mathrm{yr}^{-1}$ within the region 0 .' 7 from the source.

To estimate the mass-loss rate, we have adopted the method of Hartigan et al. (1995). We can calculate the mass-loss rate with the equation below:

$$
\dot{M}_{J}=M_{T O T} V_{\perp} / l_{\perp}
$$

$V_{\perp}$ is the projected velocity on the plane of the sky and $l_{\perp}$ is the projected jet size of the aperture. The total mass of the jet $\mathrm{M}_{T O T}$ is estimated from the forbidden [O I] emission line with the following equation:

$$
M_{T O T}=9.61 \times 10^{-6}\left(1+\frac{n_{c}}{n_{e}}\right)\left(\frac{L_{6300}}{L_{\odot}}\right) M_{\odot}
$$

We estimate the mass-loss rate close to the star in the region within \pm 5 !! $4(\sim 750 \mathrm{AU})$ from the star. The equivalent width of [O I] $\lambda 6300$ emission line is measured to be $21 \AA$. We assume $R=8.74$ magnitude, which is the dereddened $\mathrm{R}$ magnitude of DG Tau in Hartigan et al. (1995). The line luminosity of the [O I] $\lambda 6300\left(L_{6300}\right)$ converted into solar units is $9.2 \times$ $10^{-3} L_{\odot} . \quad V_{\perp}$ is estimated to be $75 \mathrm{~km} \mathrm{~s}^{-1}$ for the measured radial velocity $120 \mathrm{~km} \mathrm{~s}^{-1}$, applying the inclination angle of $32^{\circ}$ with respect to the line of sight. For $l_{\perp}$, we adopted $6.07 \times 10^{10} \mathrm{~km}$ as the projected jet aperture size which corresponds to our slit-width of $2^{\prime \prime} .9$ at a $140 \mathrm{pc}$ distance to the Taurus molecular cloud. The FWHM of the jet measured from Dougados et al. (2000) is $\sim 0$.' $21-1^{\prime \prime} .25$ at a distance of $\sim 0$ !. $4-4^{\prime \prime}$ from the star. Agra-Amboage et al. (2011) obtained the value of $\sim 0$.' $1-0^{\prime \prime} .8$ within $\sim 1^{\prime \prime}$. 4 from the source, for the medium velocity component of the blueshifted jet. The jet width increases with distance in the vicinity of the source. We assume that the jet width in our estimation would be larger than 1 .' 25 because we estimate a massloss rate within \pm 5 "! 4 . $l_{\perp}$ of 2 .' 9 should be regarded as an upper limit. The temperature is assumed to be $8200 \mathrm{~K}$, the average temperature for the [O I] $\lambda 6300$ line (Hartigan et al. 1994) and the critical density $n_{c}$ in this temperature is $1.97 \times 10^{6} \mathrm{~cm}^{-3}$. For the electron density $n_{e}, 7 \times 10^{4} \mathrm{~cm}^{-3}$ is used. The mass-loss rate, $\dot{M}_{J}$, for the $[\mathrm{O} \mathrm{I}]$ emission line is $\sim 1.0 \times 10^{-7} M_{\odot}$ $\mathrm{yr}^{-1}$.

The $\dot{M}_{J}$ can be also estimated using the [S II] $\lambda 6731$ emission line. $M_{T O T}$ is given by:

$$
M_{T O T}=1.43 \times 10^{-3}\left(\frac{L_{6731}}{L_{\odot}}\right) M_{\odot}
$$

The equivalent width of the $[\mathrm{S} \mathrm{II}] \lambda 6731$ line is $\sim 4.5 \AA$. The $\dot{M}_{J}$ from $[\mathrm{S}$ II $]$ is $1.1 \times 10^{-7} M_{\odot} \mathrm{yr}^{-1}$, which is almost the same as that of [O I] $\lambda 6300$. The mass-loss rates of $[\mathrm{O} \mathrm{I}] \lambda 6300$ and $[\mathrm{S} \mathrm{II}] \lambda 6731$ lines are a factor of 3 lower than the value of Hartigan et al. (1995) and is $3-7$ times larger than those estimated by LavalleyFouquet et al. (2000) and Agra-Amboage et al. (2011). The calculated rate can differ with time because the measured line intensities and velocities of the jet could change due to the variability of the accretion/outflow rate of YSO. The rate becomes about two times larger if we replace the jet aperture size in our calculation with the value in Hartigan et al. (1995), which is $1^{\prime \prime} .5$. The difference in spatial resolution between ours and higher-resolution (e.g., Agra-Amboage et al. 2011) observations cloud cause the difference in the mass-loss rate. 


\section{SUMMARY}

We have investigated the kinematics and physical characteristics of $\mathrm{HH} 158$ and $\mathrm{HH} 702$ from the optical long slit spectroscopy. From the analysis through the PVDs and line profiles depending on the distance from the source, the peak velocity varies in the range of -50 to $-250 \mathrm{~km} \mathrm{~s}^{-1}$ and the line width was measured to 150 to $250 \mathrm{~km} \mathrm{~s}^{-1}$. The proper motions were measured for knot B1, B2 and C of HH 158: 0.'230, 0.'272, and 0". 180 $\mathrm{yr}^{-1}$ for the knots, respectively.

From our analysis of the line ratios, we find that the physical properties $\left(n_{e}, x_{e}, T_{e}\right)$ are changing along the jet from the proximity to the star to knot $\mathrm{C}$ at $14^{\prime \prime}$ : (1) $n_{e}$ varies from an order of $10^{4}$ to $10^{2} \mathrm{~cm}^{-3},(2) x_{e}$ varies from $\sim 0$ to 0.4 at $14^{\prime \prime},(3) T_{e}$ varies from 15,000 $\mathrm{K}$ to $\sim 5,000 \mathrm{~K}$. It is noticeable that the line ratios and physical parameters of $\mathrm{HH} 702$ do not show much difference from those obtained at knot C of $\mathrm{HH} 158$. The mass-loss rate $\left(\dot{M}_{J}\right)$ is estimated to be $\sim 10^{-7} M_{\odot}$ $\mathrm{yr}^{-1}$, similar to values obtained in previous studies.

\section{ACKNOWLEDGMENTS}

We thank the staff of the BOAO (Bohyunsan Optical Astronomy Observatory) for their support during the observing runs.

\section{REFERENCES}

Agra-Amboage, V. 2009, Observations of the Inner Regions of Winds around Young T Tauri Type Stars, Ph.D. Thesis., Joseph Fourier University

Agra-Amboage, V., Dougados, C., Cabrit, S., \& Reunanen, J. 2011, Sub-Arcsecond [Fe II] Spectro-Imaging of the DG Tauri Jet. Periodic Bubbles and a Dusty Disk Wind?, A\&A, 532, A59

Bacciotti, F., Chiuderi, C., \& Oliva, E. 1995, The Structure of Optical Stellar Jets: a Phenomenological Analysis., A\&A, 296, 185

Bacciotti, F., \& Eislöffel, J. 1999, Ionization and Density along the Beams of Herbig-Haro Jets, A\&A, 342, 717

Bacciotti, F., Mundt, R., Ray, T. P., et al. 2000, Hubble Space Telescope STIS Spectroscopy of the Optical Outflow from DG Tauri: Structure and Kinematics on Subarcsecond Scales, ApJ, 537, L49

Bacciotti, F., Ray, T. P., Mundt, R., Eislöffel, J., \& Solf, J. 2002, Hubble Space Telescope/STIS Spectroscopy of the Optical Outflow from DG Tauri: Indications for Rotation in the Initial Jet Channel, ApJ, 576, 222

Bally, J., \& Devine, D. 1994, A Parsec-Scale 'Superjet' and Quasi-Periodic Structure in the HH 34 Outflow?, ApJ, 428, L65

Bally, J., Devine, D., Fesen, R. A., \& Lane, A. P. 1995, Twin Herbig-Haro Jets and Molecular Outflows in L1228, ApJ, 454,345

Beck, T. L., Bary, J. S., \& McGregor, P. J. 2010, Spatially Extended Brackett Gamma Emission in the Environments of Young Stars, ApJ, 722, 1360

Beckwith, S. V. W., Sargent, A. I., Chini, R. S., \& Guesten, R. 1990, A Survey for Circumstellar Disks around Young Stellar Objects, AJ, 99, 924

Dougados, C., Cabrit, S., Lavalley, C., \& Ménard, F. 2000, T Tauri Stars Microjets Resolved by Adaptive Optics, A\&A, 357, L61
Eislöffel, J., \& Mundt, R. 1998, Imaging and Kinematic Studies of Young Stellar Object Jets in Taurus, AJ, 115, 1554

Hamann, F. 1994, Emission-Line studies of young stars. 4: The optical Forbidden Lines, ApJS, 93, 485

Hartigan, P., Morse, J. A., \& Raymond, J. 1994, MassLoss Rates, Ionization Fractions, Shock Velocities, and Magnetic Fields of Stellar Jets, ApJ, 436, 125

Hartigan, P., Edwards, S., \& Ghandour, L. 1995, Disk Accretion and Mass Loss from Young Stars, ApJ, 452, 736

Hartigan, P. 2003, Shock Waves in Outflows from Young Stars, Ap\&SS, 287, 111

Hartmann, L., \& Raymond, J. C. 1989, Wind-Disk Shocks around T Tauri Stars, ApJ, 337, 903

Hirth, G. A., Mundt, R., \& Solf, J. 1997, Spatial and kinematic Properties of the Forbidden Emission Line Region of T Tauri Stars, A\&AS, 126, 437

Kwan, J., \& Tademaru, E. 1995, Disk Winds from T Tauri Stars, ApJ, 454, 382

Lavalley, C., Cabrit, S., Dougados, C., Ferruit, P., \& Bacon, R. 1997, Sub-Arcsecond Morphology and Kinematics of the DG Tauri Jet in the [O I] $\lambda 6300$ Line, A\&A, 327, 671

Lavalley-Fouquet, C., Cabrit, S., \& Dougados, C. 2000, DG Tau: A Shocking Jet, A\&A, 356, L41

Lynch, C., Mutel, R. L., Güdel, M., et al. 2013, Very Large Array Observations of DG Tau's Radio Jet: A Highly Collimated Thermal Outflow, ApJ, 766, 53

Maurri, L., Bacciotti, F., Podio, L., et al. 2014, Physical Properties of the Jet from DG Tauri on Sub-Arcsecond Scales with HST/STIS, A\&A, 565, A110

McGroarty, F., \& Ray, T. P. 2004, Classical T Tauri Stars as Sources of Parsec-Scale Optical Outflows, A\&A, 420, 975

McGroarty, F., Ray, T. P., \& Froebrich, D. 2007, Proper Motion Studies of Outflows from Classical T Tauri Stars, A\&A, 467, 1197

Mundt, R., Brugel, E. W., \& Bührke, T. 1987, Jets from Young Stars - CCD Imaging, Long-Slit Spectroscopy, and Interpretation of Existing Data, ApJ, 319, 275

Mundt, R., \& Fried, J. W. 1983, Jets from Young Stars, ApJ, 274, L83

Ogura, K. 1995, Giant Bow Shock Pairs Associated with Herbig-Haro Jets, ApJ, 450, L23

Osterbrock, D. E. 1989, Astrophysics of Gaseous Nebulae and Active Galactic Nuclei, (Mill Valley, CA: University Science Books)

Pyo, T.-S., Hayashi, M., Kobayashi, N., et al. 2002, Velocity-Resolved [Fe II] Line Spectroscopy of L1551 IRS 5: A Partially Ionized Wind under Collimation around an Ionized Fast Jet, ApJ, 570, 724

Pyo, T.-S., Hayashi, M., Kobayashi, N., et al. 2006, Adaptive Optics Spectroscopy of the [Fe II] Outflows from HL Tauri and RW Aurigae, ApJ, 649, 836

Pyo, T.-S., Kobayashi, N., Hayashi, M., et al. 2003, Adaptive Optics Spectroscopy of the [Fe II] Outflow from DG Tauri, ApJ, 590, 340

Ray, T. P. 1987, CCD Observations of Jets from Young Stars, A\&A, 171, 145

Raga, A. C., Binette, L., Canto, J., \& Calvet, N. 1990, Stellar Jets with Intrinsically Variable Sources, ApJ, 364, 601

Raga, A., Cabrit, S., Dougados, C., \& Lavalley, C. 2001, A Precessing, Variable Velocity Jet Model for DG Tauri, A\&A, 367, 959

Raga, A. C., \& Kofman, L. 1992, Knots in Stellar Jets from 
Time-Dependent Sources, ApJ, 386, 222

Reipurth, B., Bally, J., Aspin, C., et al. 2013, HH 222: A Giant Herbig-Haro Flow from the Quadruple System V380 Ori, AJ, 146, 118

Rodríguez, L. F., González, R. F., Raga, A. C., et al. 2012, Radio Continuum Emission from Knots in the DG Tauri Jet, A\&A, 537, A123

Solf, J., \& Böhm, K. H. 1993, High-Resolution Long-Slit Spectral Imaging of the Mass Outflows in the Immediate
Vicinity of DG Tauri, ApJ, 410, L31

Sun, K.-F., Yang, J., Luo, S.-G., et al. 2003, Large-Scale Distribution of Herbig-Haro Objects in Taurus, ChJAA, 3,458

Whelan, E. T., Ray, T. P., \& Davis, C. J. 2004, Paschen $\beta$ Emission as a Tracer of Outflow Activity from TTauri Stars, as Compared to Optical Forbidden Emission, A\&A, 417, 247 